\title{
Clinicopathological characteristics in women with breast cancer in extreme ages in Greece: A single centre experience
}

\author{
Charalampos Karachalios ${ }^{1 *}$, Evangelos Karamitrousis ${ }^{2}$, Panagiotis Daskalakis ${ }^{1}$ \\ ${ }^{1}$ Breast Center, "Helena Venizelou" Maternity Hospital, Athens, Greece \\ 2Medical Oncology Department, Aristotle University of Thessaloniki, \\ Papageorgiou General Hospital, Greece
}

Received 26 April 2021; Accepted 29 September 2021

\begin{abstract}
Introduction: Breast cancer is the most common malignancy in Greek women. The highest incidence occurs in patients aged 40-70 years. However, the emergence of the disease in women under 40 and over 80 years old is a significant problem in everyday practice, because of the diagnostic and therapeutic challenges for these age groups. The objective of this study is to investigate clinicopathological characteristics of Greek women under 40 and over 80 diagnosed with breast cancer during 2019-2020 in a specialized Breast Cancer Surgical Centre, as well as the pathological characteristics of their disease and the therapeutic measures applied to these women. Aim of the study: The aim of this study is to compare clinical and pathological characteristics of breast cancer in a group of Greek premenopausal women younger than 40 years of age with a group of elderly women older than 80 years old, who were treated in a surgical breast centre in Greece during the last two years.

Patients and Methods: During the last 2 years, 21 women under 40 and 22 women older than 80 were diagnosed with breast cancer in our Centre.

Results: Invasive ductal adenocarcinoma was the most frequent histological type for both Groups: Most younger women underwent surgery followed by chemotherapy, while the majority of older patients received hormonal therapy after surgery.

Conclusions: There were no statistically significant differences between women under 40 and women over 80 in the study sample regarding their clinicopathological characteristics. However, younger women underwent more therapeutic modalities (i.e.adjuvant treatments in addition to surgery) than older ones.
\end{abstract}

Keywords: Breast • cancer • elderly • young women • Greek

\section{List of abbreviations}

AYA: Adolescents and Young Adults

US(A): United States (of America)

EUSOMA: European Society of Breast Cancer Specialists

AJCC: American Joint Committee on Cancer

$\mathrm{GnRH}$ : Gonadotropin-releasing hormone

HR: hormone receptors

CMF: cyclophosphamide, methotrexate and fluorouracil

\section{Introduction}

Breast cancer is the most common malignancy, not only worldwide, but also in women across Greece. According to recent statistical data from the Global Cancer Observatory (GLOBOCAN), it is estimated that there were approximately 7,772 new breast cancer cases and almost 2,333 deaths from breast cancer for females of all ages in 2020 in Greece ${ }^{[1]}$. During the years 2012-2016 in the USA, the median age of patients at the time of breast cancer diagnosis was $62^{[2]}$. Most researchers define breast cancer cases in patients younger than 40 years old as 'early onset disease'[3]. At the other end of the age spectrum, women diagnosed with breast cancer after 80 represent the 'older extremity' of the disease.

In women younger than 45 , breast cancer is the leading cause of death related to cancer. The highest age threshold of a 'young woman' in breast oncology settings varies from 35 to 45 years. However, as stated above, 
most studies regard breast cancer occurring in women younger than 40 as 'very early onset disease'[4]. Breast cancer is the most common cancer among adolescents and young adults (AYA) aged 15 to 39 years in the United States. Approximately 11,000 AYA females were diagnosed with invasive breast cancer in 2013 in the US and approximately 1,000 died of the disease. From 2000 to 2014 in the US, $5.6 \%$ of all invasive breast cancer cases were diagnosed in women younger than $40^{[5]}$. Survival rates are lower for young women compared to older ones ${ }^{[6,7]}$, as the former females have shown worse clinical outcomes, implementation of more extensive treatment modalities and more long-term treatment complications than the latter ones ${ }^{[3,8]}$, as well as a predisposition to secondary breast or ovarian tumours ${ }^{[9]}$. Younger women tend to present at later stages and with larger and more aggressive tumours ${ }^{[7,10]}$.

On the contrary, more than half of all breast cancer cases occur in women older than 60 years of age ${ }^{[14]}$. The age of 70 is commonly adopted as the threshold value for elderly patients ${ }^{[15]}$. Older adults are the fastest growing part of the population in the industrialised countries ${ }^{[16]}$. This proportion will increase as the life expectancy of the population improves ${ }^{[17]}$. Although the average life expectancy of females older than 65 years is 86.6 years, $25 \%$ will live to more than 90 years old and $10 \%$ will live more than $95^{[14]}$. The number of women older than 75 totaled 11.5 million in 2012 and is projected to reach 25 million by $2040^{[18]}$. Additionally, it is estimated that between 2000 and 2050 the population of women aged over 80 will have more than tripled in developed countries $^{[19]}$. Luckily, most breast cancers in older women are identified at an early, treatable stage, and the majority of women diagnosed with early stage breast cancer enjoy prolonged disease-free survival ${ }^{[24]}$. Clinicians who manage elderly women with breast cancer should take into consideration their functional status, comorbidities, clinical stages, biological characteristics of the malignant neoplasm, and life expectancy. Therefore, they should choose the most appropriate adjuvant therapeutic modalities, such as chemotherapy, radiation therapy, and endocrine treatment, to maximise the efficacy of the cure and maintain disease-free status, while, at the same time, minimising interruptions in quality of life and unexpected early death ${ }^{[25]}$.

\section{Patients and Methods}

This retrospective study was performed at the Breast Centre of 'Elena Venizelou' Athens General Maternity Hospital and approved by the Institutional Ethics Committee for Research involving human subjects.
The study has followed every standard set by the 1964 Declaration of Helsinki of the World Medical Association General Assembly.

A review of our centre's medical records database was undertaken to identify all Greek patients younger than 40 and older than 80 years old who were treated for primary breast cancer from 1 January 2019 to 31 December 2020. Women with a previous history of breast malignancy, metastatic breast cancer or bilateral breast malignant neoplasms were excluded. These patients were subdivided into two groups. The first group (Group A) encompassed women less than 40 years of age. The second group (Group B) consisted of patients more than 80 years of age. All were treated according to the European Society of Breast Cancer Specialists (EUSOMA) guidelines under the joint care of a breast surgeon and a medical oncologist. Clinical, pathological, and treatment variables were collected. Pathological staging was performed using the American Joint Committee on Cancer (AJCC) staging guidelines. Data included the histological type of tumour, histological grade, tumour size, lymph nodes status, tumour stage, hormonal receptor status, type of surgery, systemic chemotherapy, radiation therapy, and endocrine treatment. All pathology specimens were reviewed at our institution's Pathology Department. Estrogen (ER) and progesterone (PR) receptors were defined as positive if tumour cell nuclei staining is $\geq 1 \%$.

The statistical analysis was undertaken by a biostatistician using SPSS ${ }^{\mathrm{TM}}$ statistical software, version 25 (IBM Corporation, Armonk, New York, United States of America). On comparison of patient groups, Fisher's exact test and the chi-square test for trend were used. A $p$-value less than 0.05 was considered significant.

\section{Results}

The study sample consisted of 21 patients (48.8\%) less than 40 years of age and 22 women (51.2\%) over 80 years of age. The age range was 30 to 39 and 81 to 89 years of age for Groups A and B, respectively. Mean age was 34.8 and 83.6 years old for Groups A and B, respectively. As shown in Tables 1 and 2, there are no statistically significant differences between the above groups regarding histological type of tumour, tumour grade, tumour size, lymph node status, TNM tumour stage, hormonal receptors status, type of surgery, or implemented therapeutic modalities. Only a minority of Group B women received no treatment, while every woman in Group A was treated. The most common histological type was ductal invasive carcinoma for both groups. More patients in both groups had a grade 3 tumour 
Table 1: Clinicopathological characteristics of the study population.

\begin{tabular}{|c|c|c|c|c|}
\hline & \multicolumn{3}{|c|}{ Age group (years) } & \multirow{2}{*}{$\mathbf{P}$} \\
\hline & $<40$ & $>80$ & Total & \\
\hline \multicolumn{5}{|c|}{ Histological Type, n (\%) } \\
\hline Ductal invasive & $12(57.14 \%)$ & $13(59.09 \%)$ & $25(58.14 \%)$ & \multirow{3}{*}{0.1472} \\
\hline Lobular invasive & $3(14.29 \%)$ & $2(9.09 \%)$ & $5(11.63 \%)$ & \\
\hline Other & $6(28.57 \%)$ & $7(31.82 \%)$ & $13(30.23 \%)$ & \\
\hline \multicolumn{5}{|c|}{ Tumor Size (T), n (\%) } \\
\hline $\mathrm{T} \leq 2 \mathrm{~cm}$ & 7 (33.33\%) & $9(40.90 \%)$ & $16(37.21 \%)$ & \multirow[t]{2}{*}{0.7546} \\
\hline $\mathrm{T}>2 \mathrm{~cm}$ & $14(66.67 \%)$ & $13(59.10 \%)$ & 27 (62.79\%) & \\
\hline \multicolumn{5}{|c|}{ Positive lymph nodes, n (\%) } \\
\hline 0 lymph nodes & $11(52.38 \%)$ & $12(54.54 \%)$ & $23(53.49 \%)$ & \multirow{3}{*}{0.8948} \\
\hline 1-3 lymph nodes & $6(28.57 \%)$ & $5(22.73 \%)$ & $11(25.58 \%)$ & \\
\hline$\geq 4$ lymph nodes & $4(19.05 \%)$ & $5(22.73 \%)$ & $9(20.93 \%)$ & \\
\hline \multicolumn{5}{|c|}{ TNM Stage, n (\%) } \\
\hline 1 & $4(19.05 \%)$ & $4(18.18 \%)$ & $8(18.61 \%)$ & \multirow{3}{*}{0.4609} \\
\hline$\|$ & $13(61.90 \%)$ & $11(50.00 \%)$ & $24(55.81 \%)$ & \\
\hline III & $4(19.05 \%)$ & $7(31.82 \%)$ & $11(25.58 \%)$ & \\
\hline \multicolumn{5}{|c|}{ Hormone receptor status, n (\%) } \\
\hline $\mathrm{ER}+/ \mathrm{PR}+$ & $10(47.62 \%)$ & $11(50.00 \%)$ & $21(48.84 \%)$ & \multirow{4}{*}{0.9571} \\
\hline ER-/PR- & $6(28.57 \%)$ & $5(22.73 \%)$ & $11(25.58 \%)$ & \\
\hline $\mathrm{ER}+/ \mathrm{PR}-$ & $3(14.28 \%)$ & $3(13.64 \%)$ & $6(13.95 \%)$ & \\
\hline ER-/PR+ & $2(9.53 \%)$ & $3(13.64 \%)$ & $5(11.63 \%)$ & \\
\hline \multicolumn{5}{|c|}{ Histological Grade, n (\%) } \\
\hline 1 & 3 (14.29\%) & $2(9.09 \%)$ & $5(11.63 \%)$ & \multirow{3}{*}{0.4754} \\
\hline$\|$ & $5(23.81 \%)$ & $9(40.91 \%)$ & $14(32.56 \%)$ & \\
\hline III & $13(61.90 \%)$ & $11(50.00 \%)$ & $24(55.81 \%)$ & \\
\hline
\end{tabular}

Table 2: Therapeutic interventions in the study population.

\begin{tabular}{cccc}
\hline & Extend of surgical procedure, n (\%) & \\
\hline Lumpectomy & $8(38.10 \%)$ & $5(22.73 \%)$ & $13(30.23 \%)$ \\
Quadrantectomy & $4(19.05 \%)$ & $4(18.18 \%)$ & $7(16.6 \%)$ \\
Simple mastectomy & $2(9.52 \%)$ & $5(22.73 \%)$ & $11(25.58 \%)$ \\
Modified radical & $7(33.33 \%)$ & $4(18.18 \%)$ & $4(9.3 \%)$ \\
mastectomy & 0 & $4(18.18 \%)$ & $21(38.89 \%)$ \\
No surgery & Adjuvant therapeutic modalities, n (\%) & $15(27.78 \%)$ \\
\hline Chemotherapy & $12(44.44 \%)$ & $9(33.33 \%)$ & $18(33.33 \%)$ \\
Radiotherapy & $10(37.04 \%)$ & $5(18.52 \%)$ & 0.0593 \\
Endocrine therapy & $5(18.52 \%)$ & $13(48.15 \%)$ & \\
\hline
\end{tabular}

size greater than $2 \mathrm{~cm}$ at the time of diagnosis, but with no positive lymph nodes. Modified radical mastectomy was more common in younger women. More patients in Group A underwent chemotherapy and radiotherapy than patients in Group B, whereas the opposite is true regarding endocrine treatment. There were no women in both groups who did not receive any treatment at all.

\section{Discussion}

Breast cancer risk and mortality among young women are expected to rise in the next decades, due to an increase in obesity and diabetes incidence ${ }^{[11]}$. Breast cancer in younger women is responsible for significant excess costs in medical treatment and decreased productivity ${ }^{[10]}$. Younger women are a heterogeneous group, at various developmental stages. Their concerns and needs differ significantly from older women, who, many times, have friends who have experienced breast cancer and for whom years of screening mammography and educational campaigns have alerted them to the possibility of breast cancer occurring ${ }^{[12]}$. Moreover, younger women face specific issues related to body image, sexuality, fertility preservation, desire for pregnancy, lactation, impact on current and future 
relationships, child care, career opportunities, and longterm toxicity potentially affecting survivorship ${ }^{[13]}$. Indeed, young women face breast cancer as an unexpected and rare event. They usually know no peers suffering from the disease, and they may not have completed their education or professional development. They also may be or may not be in a long-term relationship with a partner. If they have no children, cancer treatment may disrupt their family planning plans. If they are already parents, they may fear for their ability to successfully raise their children. Younger women perceive cancer as more threatening and report greater fear of cancer recurrence than older women ${ }^{[12]}$.

A multidisciplinary approach by breast surgical oncologists, medical oncologists with experience in treating breast cancer patients, radiation oncologists, reconstructive plastic surgeons, reproductive endocrinologists, gynaecologists specialised in managing subfertile couples, pathologists, geneticists, geriatricians, psychologists, nutritionists, breast nurses, and social workers is crucial for tailoring the therapeutic modalities chosen for individuals of these extreme age groups ${ }^{[13]}$. This holistic approach can maximise the safety and efficacy of most interventions by improving quality of life and increasing survival rates.

The behavior of breast cancer in very young women is usually more aggressive in comparison with older ones, which contributes to a poorer prognosis ${ }^{[4,}$ 29]. This partly relates to diagnostic delays and more local recurrences ${ }^{[13]}$. Furthermore, young patients not only report high rates of lymphovascular invasion and lymphocytic infiltration, but they also have tumours with negative estrogen and progesterone receptor status ${ }^{[4,}$ 29]. These characteristics have been linked to Human Epidermal growth factor Receptor-2 (HER-2)/neu overexpression, which correlates with the development of breast cancer in younger ages, more aggressive phenotypes, and more lymph node metastases ${ }^{[5,8,29]}$. However, in both age groups of women younger than 40 and older than 80 invasive ductal carcinoma is the most frequent histological type, with invasive lobular carcinoma being the second most common type ${ }^{[29]}$. The latter is also confirmed by our study.

Another important aspect of breast cancer in patients younger than 40 is that of familial breast cancer. The younger the patient, the more it seems that a BRCA1/BRCA2 deleterious mutation is responsible for her disease, especially when a positive family history accompanies a hormone-receptor negative or triple negative tumour ${ }^{[9]}$. The burden of a breast cancer diagnosis at a very young age becomes even heavier, because these women face the consideration of bilateral mastectomy and bilateral prophylactic salpingo- oophorectomy. Furthermore, these women must undergo increased surveillance for a new primary breast and/or ovarian cancer, including annual breast magnetic resonance imaging and transvaginal ultrasonography ${ }^{[6]}$. Point mutations in TP53 and PIK3CA genes have been observed in a quarter of cases ${ }^{[4]}$. A germline mutation in the TP53 gene has been linked to Li Fraumeni syndrome, an autosomal dominant genetic syndrome leading to increased rates at an earlier onset, such as sarcomas, brain malignancies, and adrenocortical carcinomas ${ }^{[13]}$. Women with high risk of breast cancer and negative BRCA1 and BRCA2 should be tested for the PALB2, CHEK2*1100delC, ATM, NBS1, RAD50, BRIP1, and $\mathrm{CDH}-1$ mutations ${ }^{[9,11]}$. Gene sets unique to breast tumours arising in younger women include those related to immune function, the mammalian target of the rapamycin/rapamycin pathway, hypoxia, BRCA1, stem cells, apoptosis, histone deacetylase, and multiple oncogenic signaling pathways, including the Myc, E2F, Ras, b-catenin, AKT, p53, PTEN, and MAP kinase pathways $^{[9]}$. Most patients in our study sample were hormone-receptor positive. Hormone-receptor negative patients were approximately even in both age groups.

Secondary prevention of breast cancer in women younger than 40 remains a complex task for clinicians. These women are not enrolled in screening programs, because breast cancer is rare in young women and mammography has a low sensitivity in dense young breasts $^{[13]}$. Thus, primary prevention campaigns should focus on lifestyle modifications, such as cessation of smoking, reduction in alcohol intake, maintenance of a healthy body weight with frequent moderate exercise, lower consumption of processed foods and red meat, and vitamin D supplementation ${ }^{[5]}$. Further modifiable risk factors include delayed childbearing, lower parity, and decreased lactation. High parity and pregnancy at an early age reduce breast cancer risk, because of reduction in the total number of menstrual cycles. Moreover, endocrine disruptors and night-shift occupation contribute to early onset breast carcinoma ${ }^{[9]}$.

The higher rate of local recurrences after breastconserving surgery in young patients should not guide clinicians to choose more aggressive treatments. Chemotherapy, targeted therapies, and endocrine treatment can impair fertility ${ }^{[13]}$. Before the initiation of any systemic therapy, all young women with breast malignancy should be offered referral to an assisted reproduction unit for either embryo or oocyte cryopreservation. Preimplantation genetic diagnosis (PGD) can aid in the selection of unaffected embryos for implantation. This strategy is an option for BRCA mutation carrier mutations or other cancer predisposition syndromes $^{[5]}$. The concomitant administration of a $\mathrm{GnRH}$ 
analogue for ovarian suppression during chemotherapy can allow fertility preservation in young patients with hormone-receptor-negative breast cancers ${ }^{[5,13]}$.

Advanced age is the major risk factor for breast cancer and is regarded as a significant independent predictor of poor breast cancer prognosis ${ }^{[20,21]}$. Nearly one third of all patients diagnosed with breast cancer are 70 years of age or older ${ }^{[20]}$. In fact, a US woman of average risk who is and has been free of breast cancer until 75 still has a $5.2 \%$ probability of developing breast cancer during her lifetime ${ }^{[18]}$. As a result, old women experience most breast cancer diagnoses and deaths ${ }^{[16]}$. Breast cancer in the elderly shows different clinicopathological features than in younger women ${ }^{[19]}$. Although these women are thought to suffer from biologically more favorable tumours, usually with clinical lymph node-negative breast cancers, more often hormone-receptor positive neoplasms, with a lower grade and a lower proliferation rate in comparison with younger ones, breast cancer is more likely to be diagnosed at an advanced stage in elderly women, who tend to experience inferior access to cancer services and treatments in comparison with younger patients ${ }^{[21-23]}$. Indeed, many patients older than 85 self-refer because screening is not provided to them. In general, the older the patients, the more likely their initial presentation of breast cancer will be a palpable mass $^{[14]}$.

Unfortunately, it has been shown that older patients are more likely to receive non-standardised care, which usually depends more on a physician's preference. The poor outcomes observed among older patients can also be attributed to various comorbidities and its associated medications. Such comorbidities can affect the mortality of older women regardless of their breast cancer or its treatment ${ }^{[26]}$. Elderly women with early stage breast cancer and comorbid conditions are likely to die from other causes rather than their operable breast cancer ${ }^{[27,28]}$. However, epidemiological studies indicate that this is untrue until the age of $85^{[19]}$. Age alone should not dictate any aspect of management for older individuals with breast cancer. All decisions should consider physiological age, estimated life expectancy, risks, benefits, treatment, tolerance, patient preferences, and potential treatment barriers ${ }^{[27]}$. Actually, underestimation of life expectancy and fitness for therapy might result in age-related undertreatment, which is itself a risk factor for breast cancer recurrence and death, and decisions regarding therapy for breast cancer should not be based on age alone ${ }^{[26,27]}$.

Indeed, some studies show that these women are more likely to be surgically overtreated and receive less adjuvant therapies ${ }^{[21]}$, while other studies emphasise the practice of monotherapy with endocrine treatments, such as tamoxifen or aromatase inhibitor alone, in HRpositive patients ${ }^{[25]}$. Although the levels of circulating estrogen in the elderly are very low, the risk of HRpositive breast cancer is higher, due to deep cellular and biological changes that the mammary glands undergo after menopause ${ }^{[19]}$. Therefore, primary endocrine therapy should be offered only to patients who refuse surgery or are unfit for it ${ }^{[15]}$. Besides, tamoxifen has been associated with thromboembolic events, endometrial cancer, and uterine sarcomas, while aromatase inhibitors can cause hot flushes, arthralgia, myalgia, and hair loss ${ }^{[14]}$. In the present study, there was a slightly statistically significant difference in endocrine treatment administered between the study groups, because older women tended to receive tamoxifen and/or aromatase inhibitors more than younger ones[13/22 (59,09\%) older women vs. $5 / 21(23,81 \%)$ ], although both groups showed similar hormone receptor expression rates, mainly because $4 / 22(18,18 \%)$ of older women received endocrine treatment as primary systemic therapy, while all younger women underwent surgical treatment.

The major factor that drives clinicians' therapeutic decisions is the presence of comorbidities, such as cerebrovascular disease, chronic renal failure, paralysis, chronic obstructive pulmonary disease, dementia, ulcers, liver disease, rheumatoid arthritis, previous malignancies, peripheral vascular disease, congestive heart failure, myocardial infarction, and diabetes ${ }^{[28]}$. Quality of life assessment in any elderly patient is imperative prior to any treatment. Therefore, a thorough geriatric assessment is crucial for individualisation of treatment decisions in elderly women. Sometimes, the real risk to the patient is not the $5 \mathrm{~mm} \mathrm{DCIS}$, but rather her hypertension or osteoporosis ${ }^{[30]}$. Not all elderly women can tolerate the negative effects of chemotherapy or even radiotherapy, and polypharmacy has been associated with increased chemotherapy toxicity. Anthracyclines and CMF are currently being replaced by anthracyclineand taxane-containing regimens ${ }^{[20,21]}$. Breast irradiation should be offered to older women with a life expectancy of more than 5 years, with large tumours, positive lymph nodes, or negative hormone receptors ${ }^{[15]}$. Moreover, elderly patients often do not comply with systemic therapies and radiation, even after surgical treatment, such as a lumpectomy ${ }^{[31]}$. In our study, an exceedingly small number of elderly women underwent endocrine treatment alone, while approximately $41 \%$ of patients older than 80 years old received chemotherapy, which is contradictory to the majority of older patients worldwide, who tend to receive endocrine treatment more than chemotherapy. Furthermore, as was stated above, there were no patients who did not receive any therapeutic intervention. 
The present study concluded that there were no statistically significant differences between women less than 40 and women more than 80 years old regarding histological type of tumours, tumour grade, tumour size, lymph node status, TNM tumour stage, hormonal receptors status, radicality of surgery, or implemented therapeutic modalities except for surgery, which is contradictory to most worldwide cases of the above age groups. The main weaknesses of the present study are the relatively small sample size, the absence of data regarding the HER2/neu status of the participants' tumours, and the lack of follow-up for these patients,

\section{References}

1] Sung, H. et al., 'Global Cancer Statistics 2020: GLOBOCAN Estimates of Incidence and Mortality Worldwide for 36 Cancers in 185 Countries', CA Cancer J Clin, 2021, https://doi.org/10.3322/ caac. 21660 .

[2] Howlader, N. et al., SEER Cancer Statistics Review, 1975-2016, 2019.

[3] Schaffar, R. et al., 'A Population-Based Cohort of Young Women Diagnosed with Breast Cancer in Geneva, Switzerland', PLoS One 14, 2019, https:// doi.org/10.1371/journal.pone.0222136.

[4] Anastasiadi, Z. et al., 'Breast Cancer in Young Women: An Overview. Updates Surg, 2017', pp. 313-317.

[5] Johnson, R.H. et al., Breast cancer in adolescents and young adults. Pediatr Blood Cancer, 2018, 65:e27397, https://doi.org/10.1002/pbc.27397,

[6] Rosenberg, S.M. et al., 'BRCA1 and BRCA2 Mutation Testing in Young Women with Breast Cancer', JAMA Oncol, no. 2, 2016, pp. 730-736, https://doi.org/10.1001/jamaoncol.2015.5941.

[7] Walsh, S.M. et al., 'Breast Cancer in Young Black Women', Br J Surg, no. 107, 2020, pp. 677-686, https://doi.org/10.1002/bjs.11401.

[8] Plichta, J.K. et al., 'Breast Cancer Tumor Histopathology, Stage at Presentation, and Treatment in the Extremes of Age', Breast Cancer Res Treat, no. 180, 2020, pp. 227-235, https://doi. org/10.1007/s10549-020-05542-4.

[9] Gewefel, H., Salhia, B., 'Breast Cancer in Adolescent and Young Adult Women', Clin Breast Cancer, no. 14, 2014, pp. 390-395.

[10] Shoemaker, M.L. et al., 'Differences in Breast Cancer Incidence among Young Women Aged 2049 Years by Stage and Tumor Characteristics, Age, Race, and Ethnicity, 2004-2013', Breast Cancer especially regarding treatment-related adverse events and treatment outcomes, such as local relapse, relapsefree survival, and overall survival. Clinicians should tailor the guidelines to their patients' individual special needs and constantly collaborate with their colleagues of other specialties for the maximisation of the therapeutic benefits for these extreme age groups.

\section{Disclaimers}

There are no conflicts of interest.
Res Treat, no. 169, 2018, pp. 595-606, https://doi. org/10.1007/s10549-018-4699-9.

[11] Desreux, J.A.C., 'Breast Cancer Screening in Young Women', Eur J Obstet Gynecol Reprod Biol, no. 230, 2018, pp. 208-211. https://doi.org/10.1016/j. ejogrb.2018.05.018.

[12] Ganz, P.A., Bower, J.E., Stanton, A.L., 'Special Issues in Younger Women with Breast Cancer', Adv Exp Med Biol, no. 862, 2015, pp. 9-21. https://doi. org/10.1007/978-3-319-16366-6_2.

[13] Rossi, L., Mazzara, C., Pagani, O., 'Diagnosis and Treatment of Breast Cancer in Young Women', Curr Treat Options Oncol, no. 20, 2018, p. 86.

[14] Varghese, F., Wong, J., 'Breast Cancer in the Elderly', Surg Clin North Am, no. 98, 2018, pp. 819-833.

[15] Giugliano, F.M. et al., 'External Radiotherapy for Breast Cancer in the Elderly', Aging Clin Exp Res, no. 29, 2017, pp. 149-157.

[16] Meresse, M. et al., 'Chemotherapy in Old Women with Breast Cancer: Is Age Still a Predictor for Under Treatment?', Breast J, no. 23, 2017, pp. 256-266, https://doi.org/10.1111/tbj.12726.

[17] Ward, S.E. et al., 'Adjuvant Chemotherapy for Breast Cancer in Older Women: An Analysis of Retrospective English Cancer Registration Data', Clin Oncol, no. 31, 2019, pp. 444-452, https://doi. org/10.1016/j.clon.2019.03.005.

[18] Lee, C.S. et al., 'Screening for Breast Cancer in Women Age 75 Years and Older', Am J Roentgenol, no. 210, 2018, pp. 256-263, https:// doi.org/10.2214/AJR.17.18705.

[19] Lodi, M et al., 'Breast Cancer in Elderly Women and Altered Clinico-Pathological Characteristics: A Systematic Review', Breast Cancer Res Treat, no. 166, 2017, pp. 657-668. 
[20] Bagegni, N.A., Peterson, L.L., 'Age-Related Disparities in Older Women with Breast Cancer', in Advances in Cancer Research, Academic Press Inc., 2020, pp. 23-56.

[21] Grumpelt, A.M. et al., 'Tumor Characteristics and Therapy of Elderly Patients with Breast Cancer', J Cancer Res Clin Oncol, no. 142, 2016, pp. 1,1091,116, https://doi.org/10.1007/s00432-015-2111-2.

[22] Herbst, A., Ingemarsson, I., 'Intermittent Versus Continuous Electronic Monitoring in Labour: A Randomised Study', BJOG An Int J Obstet Gynaecol, 1994, https://doi.org/10.1111/j.1471-0528.1994. tb13181.x

[23] Schonberg, M.A. et al., 'Developing a Patient Decision Aid for Women Aged 70 and Older with Early Stage, Estrogen Receptor Positive, HER2 Negative, Breast Cancer', J Geriatr Oncol, no. 10, 2019, pp. 980-986, https://doi.org/10.1016/j. jgo.2019.05.004.

[24] Jolly, T.A. et al., 'Adjuvant Treatment for Older Women with Invasive Breast Cancer', Women's Heal, no. 12, 2016, pp. 129-146, https://doi. org/10.2217/whe.15.92.

[25] Jeon, Y.W. et al., 'Optimal Treatment of Breast Cancer in Women Older Than 75 Years: A Korea Breast Cancer Registry Analysis', Breast Cancer Res Treat, no. 178, 2019, pp. 693-701, https://doi. org/10.1007/s10549-019-05426-2.

[26] Gal, O. et al., 'Early Breast Cancer in the Elderly: Characteristics, Therapy, and Long-Term Outcome', Oncology, no. 94, 2018, pp. 31-38, https://doi. org/10.1159/000480087.

[27] Biganzoli, L. et al., 'Management of Elderly Patients with Breast Cancer: Updated Recommendations of the International Society of Geriatric Oncology (SIOG) and European Society of Breast Cancer Specialists (EUSOMA)', Lancet Oncol, 2012, p. 13, e148-e160.

[28] Abdel-Razeq, H. et al., 'Tumor Characteristics and Treatment Outcomes of Older Patients with Breast Cancer in Jordan', BMC Womens Health, no. 20, 2020, p. 118, https://doi.org/10.1186/s12905-02000981-z.

[29] Erić, I. et al., 'Breast Cancer in Young Women: Pathologic and Immunohistochemical Features', Acta Clin Croat, no. 57, 2018, pp. 497-502, https:// doi.org/10.20471/acc.2018.57.03.13.

[30] Baban, C.K., Devane, L., Geraghty, J., 'Change of Paradigm in Treating Elderly with Breast Cancer: Are We Undertreating Elderly Patients?', Ir J Med Sci, no. 188, 2019, pp. 379-388.
[31] Agborbesong, O. et al., 'Breast Cancer Treatment in the Elderly: Do Treatment Plans That Do Not Conform to NCCN Recommendations Lead to Worse Outcomes?', American Journal of Surgery, 2020, pp. 381-384. 